\title{
Mixed Boundary-Value Analysis of Rocking Vibrations of an Elastic Strip Foundation on Elastic Soil with Saturated Substrata
}

\author{
Xiaohua Ma, ${ }^{1,2}$ Zhenyu Wang, ${ }^{3}$ Yuanqiang Cai, ${ }^{3}$ and Changjie $\mathrm{Xu}^{3}$ \\ ${ }^{1}$ Geotechnical Engineering Institute, Zhejiang University, Hangzhou 310058, China \\ ${ }^{2}$ College of Civil Engineering and Architecture, Zhejiang University, Hangzhou 310058, China \\ ${ }^{3}$ Zhejiang Geofore Geotechnical Engineering Co., Ltd., Hangzhou 310013, China
}

Correspondence should be addressed to Zhenyu Wang; wzyu@zju.edu.cn

Received 23 October 2012; Accepted 24 February 2013

Academic Editor: Juan Torregrosa

Copyright (C) 2013 Xiaohua Ma et al. This is an open access article distributed under the Creative Commons Attribution License, which permits unrestricted use, distribution, and reproduction in any medium, provided the original work is properly cited.

\begin{abstract}
The dynamic response of an elastic strip foundation lying on elastic soil with saturated substrata is greatly affected by pore pressure induced by a rocking moment. In this paper, we explore the mixed boundary-value problem of the rocking vibration of an elastic strip foundation on elastic soil with saturated substrata via Biot dynamic equations. First, the wave equations concerning both the single-phase elastic layer and the saturated half-space are solved using a Fourier integral transform technique. The dual integral equations of the rocking vibration of an elastic strip foundation are established according to the mixed boundary conditions. Finally, the relationship of the dynamic compliance coefficient with the dimensionless frequency is obtained by applying Simpson's rule to conduct numerical calculation. We also analyse the influences of the elastic layer's thickness and elastic characteristic parameters of the foundation on the rocking vibration.
\end{abstract}

\section{Introduction}

Dynamic interaction between structural foundations and the underlying soil, both in theory and in practice, is widely studied in the field of geotechnical engineering and has important implications in power machine design and fundamental analysis of foundations under seismic loads. To simplify the boundary-value problem, in the early theoretical studies, the soil under the forced vibration of the foundation was often assumed to be a single-phase linear elastic medium [1-6].

However, soil is generally a two-phase material consisting of a solid skeleton and pores, which are filled with fluid. Such materials are commonly known as poroelastic materials in mechanics literature. After Biot established a theory of propagation of elastic waves in a fluid-saturated porous solid $[7,8]$ in 1956, the research significance on vibration characteristics of foundation on saturated soil became apparent. Lin [9] studied the vertical and rocking vibrations of an elastic circular plate lying on a single-phase viscoelastic medium. Iguchi and Luco [10] studied the dynamic response of a massless flexible circular plate supported on a layered viscoelastic half-space, obtaining the vertical and rocking impedance of the flexible plate and the numerical solution of contact stress beneath the plate. Halpern and Christiano [11, 12] evaluated compliance functions for the harmonic rocking and vertical motions of rigid permeable and impermeable plates bearing on a poroelastic half-space. Kassir and $\mathrm{Xu}$ [13] studied the mixed boundary-value problem of the vibration of a rigid strip foundation on a fluid-saturated porous half-space. Jin and Liu $[14,15]$ analysed the dynamic response of a rigid disk on a saturated half-space subjected to harmonic horizontal and rocking excitation. Li [16] studied the vertical vibration of a rigid strip foundation on saturated soil. Finally, a parametric study by Ma et al. [17] examined the influences of dimensionless frequency, dynamic permeability, and Poisson's ratio on saturated soil under a rocking rigid strip footing.

Most of the results reported previously concern the dynamic interaction between the rigid structural foundation and the underlying saturated half-space. As research advances in this field of mechanics, a more realistically analytical model becomes increasingly necessary. In fact, the soil of the earth's surface, because of differences in structure and sedimentation, usually has an apparent stratification, formed naturally over the course of history. During foundation construction, 
underlying soil is routinely reinforced via a variety of methods, inevitably leading to some degree of soil stratification. In practice, the underlying soil will have different physical properties (porosity, permeability, etc.), which have a layered distribution in depth. In researching dynamic interactions of soil and a structural foundation, considering the underlying soil as a homogeneous elastic or saturated medium is not sufficiently accurate. Taking into account the presence of groundwater, the soil below the groundwater level should be considered as saturated soil and the soil above the groundwater level may be regarded as an ideal, single-phase elastic layer. As for the structural foundation, assuming it to be an elastic body is more accurate than assuming it to be a rigid body.

Based on the Biot theory of elastic waves in fluid-saturated porous medium, Philippacopoulos [18] studied the vertical vibration of a rigid circular disk resting on a saturated layered half-space. Bougacha et al. [19, 20] analysed the dynamic stiffness coefficients of rigid strip and circular foundations on a saturated layered half-space using spatially semidiscrete finite element technology. Rajapakse and Senjuntichai [21] presented an exact stiffness matrix method to evaluate the dynamic response of a multilayered poroelastic medium due to time-harmonic loads and fluid sources applied in the interior of the layered medium. Yang et al. [22] neglected the fluid inertia force exerted on the soil skeleton as proposed in the works of Zienkiewicz et al. [23] and studied the steady state response of an elastic soil layer and a saturated layered half-space. Chen [24] explored the characteristics of vertical vibration of both rigid and elastic circular plates on elastic soils with saturated substrata, utilising the Hankel transform to solve the wave equations. Furthermore, the torsional and rocking vibration characteristics of a rigid circular plate on elastic soil with saturated substrata were studied by Wang [25] and Fu [26], respectively, and the effects of the thickness of the elastic layer and the vibration frequency on the plate's dynamics were analysed. The previously listed literature reviews do not present a study of the dynamics between a vibrating elastic strip foundation and elastic soil with saturated substrata.

In this paper, a novel study is presented to make up a deficiency. The wave equations concerning both the single-phase elastic layer and the saturated half-space are solved using a Fourier integral transform technique. Then, the dual integral equations of the rocking vibration of an elastic strip foundation are established according to mixed boundary conditions. The dynamic compliance coefficient's variation curve with the dimensionless frequency is obtained by applying Simpson's rule to conduct numerical calculation, and the effects of the elastic layer's thickness and the elastic characteristic parameters of the foundation on the rocking vibration are analysed.

\section{The Dynamic Equations and Their Solutions}

Soil and water weight are ignored; the soil is considered to be isotropic and the water incompressible. This paper studies the plane strain problem for an infinite-length strip with a footing

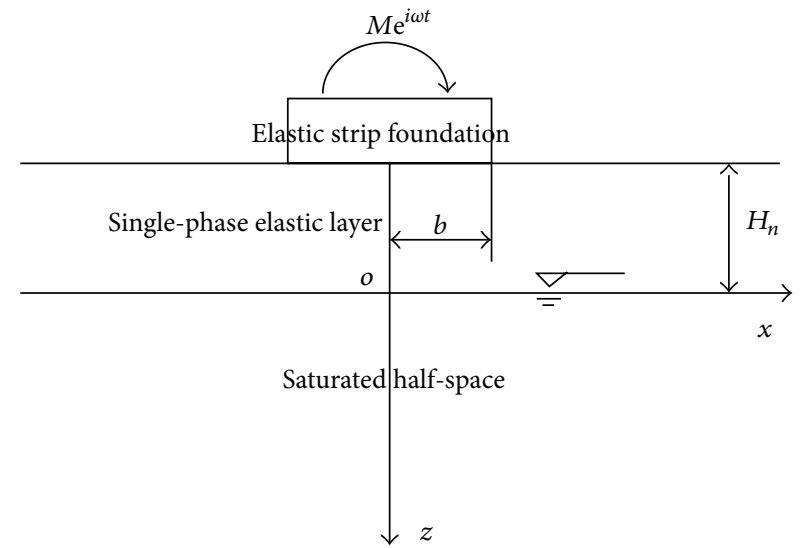

FIgURE 1: Description of the model and coordinate system.

of width $2 b$ and an elastic layer thickness of $H_{n}$. The centre of the footing is subjected to a harmonic moment force, $M e^{i \omega t}$, with $\omega$ denoting circular frequency. The horizontal direction is established as the $x$-axis and the vertical direction as the $z$ axis, and the origin of the coordinate is placed at the interface of the elastic soil and the saturated soil. The model is shown in Figure 1.

2.1. The Dynamic Equations of Elastic Layer under Plane Strain. The wave equations of the elastic layer are

$$
\begin{gathered}
\frac{\partial \sigma_{x L}}{\partial x}+\frac{\partial \tau_{x z L}}{\partial z}+\rho_{L} \frac{\partial^{2} u}{\partial t^{2}}=0 \\
\frac{\partial \tau_{x z L}}{\partial x}+\frac{\partial \sigma_{z L}}{\partial z}+\rho_{L} \frac{\partial^{2} w}{\partial t^{2}}=0
\end{gathered}
$$

where $u$ and $w$ are the horizontal and vertical displacements of the soil skeleton, respectively; $\sigma_{x L}$ and $\sigma_{z L}$ are the horizontal and vertical normal stresses, respectively; and $\rho_{L}$ is singlephase elastic soil density.

The relationship between the stress and the displacement is

$$
\begin{gathered}
\sigma_{x L}=-\frac{2 G_{L}\left(1-\mu_{L}\right)}{1-2 \mu_{L}} \frac{\partial u}{\partial x}-\frac{2 G_{L} \mu_{L}}{1-2 \mu_{L}} \frac{\partial w}{\partial z}, \\
\sigma_{z L}=-\frac{2 G_{L} \mu_{L}}{1-2 \mu_{L}} \frac{\partial u}{\partial x}-\frac{2 G_{L}\left(1-\mu_{L}\right)}{1-2 \mu_{L}} \frac{\partial w}{\partial z}, \\
\tau_{x z L}=-G_{L}\left(\frac{\partial u}{\partial z}+\frac{\partial w}{\partial x}\right),
\end{gathered}
$$

where $G_{L}$ and $\mu_{L}$ are the shear modulus and Poisson's ratio of single-phase elastic soil, respectively. 
2.2. The Dynamic Equations of Saturated Half-Space under Plane Strain. The basic dynamic equations of saturated halfspace are:

$$
\begin{gathered}
\frac{\partial \sigma_{x}^{\prime}}{\partial x}+\frac{\partial \tau_{x z}}{\partial z}+\frac{\partial p_{f}}{\partial x}+\rho \frac{\partial^{2} u}{\partial t^{2}}+\rho_{f} \frac{\partial^{2} w_{x}}{\partial t^{2}}=0 \\
\frac{\partial \tau_{x z}}{\partial x}+\frac{\partial \sigma_{z}^{\prime}}{\partial z}+\frac{\partial p_{f}}{\partial z}+\rho \frac{\partial^{2} w}{\partial t^{2}}+\rho_{f} \frac{\partial^{2} w_{z}}{\partial t^{2}}=0 \\
-\frac{\partial p_{f}}{\partial x}=\frac{\rho_{f} g}{k_{d}} \frac{\partial w_{x}}{\partial t}+\rho_{f} \frac{\partial^{2} u}{\partial t^{2}}+\frac{\rho_{f}}{n} \frac{\partial^{2} w_{x}}{\partial t^{2}} \\
-\frac{\partial p_{f}}{\partial z}=\frac{\rho_{f} g}{k_{d}} \frac{\partial w_{z}}{\partial t}+\rho_{f} \frac{\partial^{2} w}{\partial t^{2}}+\frac{\rho_{f}}{n} \frac{\partial^{2} w_{z}}{\partial t^{2}} \\
-\frac{\partial^{2} w_{x}}{\partial x \partial t}-\frac{\partial^{2} w_{z}}{\partial z \partial t}=\frac{\partial^{2} u}{\partial x \partial t}+\frac{\partial^{2} w}{\partial z \partial t}
\end{gathered}
$$

where $u$ and $w$ are the horizontal and vertical displacements of the soil skeleton, respectively; $w_{x}$ and $w_{z}$ are the horizontal and vertical displacements of water relative to the soil skeleton; $\sigma_{x}^{\prime}$ and $\sigma_{z}^{\prime}$ are the horizontal and vertical effective normal stresses, respectively; $p_{f}$ is the excess pore pressure; $\rho$ is the mass density of the saturated soil with $\rho=(1-n) \rho_{s}+n \rho_{f}$; $\rho_{s}$ and $\rho_{f}$ are the densities of the soil and water, respectively; and $n$ is the porosity of the saturated soil.

The equations of stress and displacement are

$$
\begin{gathered}
\partial \sigma_{x}^{\prime}=-\frac{2 G(1-\mu)}{1-2 \mu} \frac{\partial u}{\partial x}-\frac{2 G \mu}{1-2 \mu} \frac{\partial w}{\partial z} \\
\partial \sigma_{z}^{\prime}=-\frac{2 G \mu}{1-2 \mu} \frac{\partial u}{\partial x}-\frac{2 G(1-\mu)}{1-2 \mu} \frac{\partial w}{\partial z} \\
\tau_{x z}=-G\left(\frac{\partial u}{\partial z}+\frac{\partial w}{\partial x}\right)
\end{gathered}
$$

where $G$ and $\mu$ are the shear modulus and Poisson's ratio of the saturated soil, respectively.

2.3. The Solutions of the Dynamic Equations. For a simple harmonic load, the displacement, stress, and excess pore pressure may be expressed as

$$
\begin{gathered}
u=\bar{u} e^{i \omega t}, \\
w=\bar{w} e^{i \omega t}, \\
w_{x}=\bar{w}_{x} e^{i \omega t}, \\
w_{z}=\bar{w}_{z} e^{i \omega t}, \\
\sigma_{x L}=\bar{\sigma}_{x L} e^{i \omega t},
\end{gathered}
$$

$$
\begin{aligned}
\tau_{x z L} & =\bar{\tau}_{x z L} e^{i \omega t}, \\
\sigma_{z L} & =\bar{\sigma}_{z L} e^{i \omega t}, \\
\sigma_{x}^{\prime} & =\bar{\sigma}_{x} e^{i \omega t}, \\
\tau_{x z} & =\bar{\tau}_{x z} e^{i \omega t}, \\
\sigma_{z}^{\prime} & =\bar{\sigma}_{z}^{\prime} e^{i \omega t}, \\
p_{f} & =\bar{p}_{f} e^{i \omega t} .
\end{aligned}
$$

Here, $\omega$ is the circular frequency. The Fourier transform can be written as:

$$
\phi^{*}=\Re \phi=\int_{-\infty}^{\infty} \phi e^{i \zeta x} d x
$$

Combining (1a)-(1b) and (2a)-(2c) and utilising the Fourier transform, we can obtain the solutions of the singlephase elastic layer as follows:

$$
\begin{aligned}
i \bar{u}^{*}(\zeta, z)= & -A_{1} \zeta H_{L} e^{q_{L} z}-\frac{A_{2} e^{F_{L} z}}{\zeta}-B_{1} \zeta H_{L} e^{-q_{L} z} \\
& -\frac{B_{2} e^{-F_{L} z}}{\zeta}, \\
\bar{w}^{*}(\zeta, z)= & A_{1} q_{L} H_{L} e^{q_{L} z}+\frac{A_{2} e^{F_{L} z}}{F_{L}}-B_{1} q_{L} H_{L} e^{-q_{L} z} \\
& -\frac{B_{2} e^{-F_{L} z}}{F_{L}}, \\
& \bar{e}^{*}(\zeta, z)=A_{1} e^{q_{L} z}+B_{1} e^{-q_{L} z} \\
& 2 A_{1} \zeta q_{L} H_{L} G_{L} e^{q_{L} z}-2 B_{1} \zeta q_{L} H_{L} G_{L} e^{-q_{L} z} \\
& +A_{2}\left(\frac{F_{L}}{\zeta}+\frac{\zeta}{F_{L}}\right) G_{L} e^{F_{L} z} \\
& +B_{2}\left(\frac{F_{L}}{\zeta}+\frac{\zeta}{F_{L}}\right) G_{L} e^{-F_{L} z} \\
\sigma_{z L}^{*}(\zeta, z)=- & 2 G_{L}\left[A_{1}\left(q_{L}^{2} H_{L}+\frac{\mu_{L}}{1-2 \mu_{L}}\right) e^{-q_{L} z}\right. \\
& \left.\left.+B_{2} e^{-F_{L} z}\right], \frac{\mu_{L}}{1-2 \mu_{L}}\right) e^{q_{L} z}+A_{2} e^{F_{L} z} \\
& \\
& \\
& \\
&
\end{aligned}
$$


where

$$
\begin{gathered}
\bar{e}=\frac{\partial \bar{u}}{\partial x}+\frac{\partial \bar{w}}{\partial z}, \\
F_{L}^{2}=\zeta^{2}-\frac{\omega^{2}}{G_{L}} \rho_{L}, \\
D_{L}=-\frac{\omega^{2}\left(1-2 \mu_{L}\right) \rho_{L}}{2 G_{L}\left(1-\mu_{L}\right)}, \\
q_{L}^{2}=\zeta^{2}+D_{L}, \\
H_{L}=\frac{1}{q_{L}^{2}-\zeta^{2}}=\frac{1}{D_{L}} .
\end{gathered}
$$

Similarly, combining (3a)-(3e) and (4a)-(4c) and utilising the Fourier transform, we obtain the solutions of the saturated half-space as follows:

$$
\begin{gathered}
i \bar{u}^{*}(\zeta, z)=-A_{0} \zeta H e^{-q z}-B_{0} \zeta N e^{-\zeta z}-\frac{C_{0} e^{-F z}}{\zeta}, \\
\bar{w}^{*}(\zeta, z)=-A_{0} q H e^{-q z}-B_{0} \zeta N e^{-\zeta z}-\frac{C_{0} e^{-F z}}{F}, \\
\bar{p}_{f}^{*}(\zeta, z)=A_{0} E e^{-q z}+B_{0} e^{-\zeta z} \\
\bar{e}^{*}(\zeta, z)=A_{0} e^{-q z}, \\
i \bar{\tau}_{x z}^{*}(\zeta, z)=-2 A_{0} \zeta q H G e^{-q z}-2 B_{0} \zeta^{2} N G e^{-\zeta z} \\
-C_{0}\left(\frac{F}{\zeta}+\frac{\zeta}{F}\right) G e^{-F z}, \\
\bar{\sigma}_{z}^{*}(\zeta, z)=-2 G[ \\
{\left[A_{0}\left(q^{2} H+\frac{\mu}{1-2 \mu}\right) e^{-q z}+B_{0} \zeta^{2} N e^{-\zeta z}\right.} \\
\left.+C_{0} e^{-F z}\right]+A_{0} E e^{-q z}+B_{0} e^{-\zeta z},
\end{gathered}
$$

where

$$
\begin{gathered}
c=\frac{n k_{d} \omega}{g n i-k_{d} \omega}, \\
\bar{e}=\frac{\partial \bar{u}}{\partial x}+\frac{\partial \bar{w}}{\partial z}, \\
F^{2}=\zeta^{2}-\frac{\omega^{2}}{G}\left(c \rho_{f}+\rho\right), \\
E=\frac{\omega^{2} \rho_{f}(c+1)}{D c}, \\
D=\frac{\omega^{2}(1-2 \mu)\left(2 c \rho_{f}+\rho_{f}-c \rho\right)}{2 c G(1-\mu)},
\end{gathered}
$$

$$
\begin{gathered}
q^{2}=\zeta^{2}+D, \\
H=\frac{1}{q^{2}-\zeta^{2}}=\frac{1}{D}, \\
N=\frac{c+1}{G\left(\zeta^{2}-F^{2}\right)}=\frac{c+1}{\omega^{2}\left(c \rho_{f}+\rho\right)} .
\end{gathered}
$$

The transformation as shown in the following is introduced:

$$
\begin{array}{ll}
A_{1}=\frac{1}{2}\left(\bar{A}_{1}+\bar{B}_{1}\right), & B_{1}=\frac{1}{2}\left(\bar{A}_{1}-\bar{B}_{1}\right), \\
A_{2}=\frac{1}{2}\left(\bar{A}_{2}+\bar{B}_{2}\right), & B_{2}=\frac{1}{2}\left(\bar{A}_{2}-\bar{B}_{2}\right) .
\end{array}
$$

Equations (7a)-(7e) can be further transformed as below:

$$
\begin{aligned}
i \bar{u}^{*}(\zeta, z)= & -\bar{A}_{1} \zeta H_{L} \operatorname{ch} q_{L} z-\frac{\bar{A}_{2} \operatorname{ch} F_{L} z}{\zeta}-\bar{B}_{1} \zeta H_{L} \operatorname{sh} q_{L} z \\
& -\frac{\bar{B}_{2} \operatorname{sh} F_{L} z}{\zeta},
\end{aligned}
$$

$$
\begin{aligned}
\bar{w}^{*}(\zeta, z)= & \bar{A}_{1} q_{L} H_{L} \operatorname{sh} q_{L} z+\frac{\bar{A}_{2} \operatorname{sh} F_{L} z}{F_{L}}+\bar{B}_{1} q_{L} H_{L} \operatorname{ch} q_{L} z \\
& +\frac{\bar{B}_{2} \operatorname{ch} F_{L} z}{F_{L}}
\end{aligned}
$$$$
\bar{e}^{*}(\zeta, z)=\bar{A}_{1} \operatorname{ch} q_{L} z+\bar{B}_{1} \operatorname{sh} q_{L} z,
$$

$i \bar{\tau}_{x z L}^{*}(\zeta, z)=2 \bar{A}_{1} \zeta q_{L} H_{L} G_{L} \operatorname{sh} q_{L} z+\bar{A}_{2}\left(\frac{F_{L}}{\zeta}+\frac{\zeta}{F_{L}}\right) G_{L} \operatorname{sh} F_{L} z$

$$
\begin{aligned}
& +2 \bar{B}_{1} \zeta q_{L} H_{L} G_{L} \operatorname{ch} q_{L} z \\
& +\bar{B}_{2}\left(\frac{F_{L}}{\zeta}+\frac{\zeta}{F_{L}}\right) G_{L} \operatorname{ch} F_{L} z \\
\sigma_{z L}^{*}(\zeta, z)=-2 G_{L}[ & \bar{A}_{1}\left(q_{L}^{2} H_{L}+\frac{\mu_{L}}{1-2 \mu_{L}}\right) \operatorname{ch} q_{L} z \\
& +\bar{A}_{2} \operatorname{ch} F_{L} z+\bar{B}_{1} \\
& \times\left(q_{L}^{2} H_{L}+\frac{\mu_{L}}{1-2 \mu_{L}}\right) \operatorname{sh} q_{L} z \\
& \left.+\bar{B}_{2} \operatorname{sh} F_{L} z\right] .
\end{aligned}
$$




\section{The Mixed Boundary-Value Problem of the Rocking Vibration of an Elastic Strip Foundation on Elastic Soil with Saturated Substrata and Boundary Conditions}

It is assumed that the contact between the elastic strip foundation and the saturated soil is smooth and that the surface of the saturated soil is pervious. The boundary conditions are expressed as

$$
\begin{gathered}
\bar{w}(x, 0)=x \phi-\bar{\Delta}(x) \quad|x| \leq b, \\
\bar{\tau}_{x z L}\left(x,-H_{n}\right)=0 \quad-\infty<x<\infty, \\
{[\bar{w}(x, 0)]_{L}=\bar{w}(x, 0) \quad-\infty<x<\infty,} \\
{[\bar{u}(x, 0)]_{L}=\bar{u}(x, 0) \quad-\infty<x<\infty,} \\
\bar{\tau}_{x z L}(\zeta, 0)=\bar{\tau}_{x z}(\zeta, 0) \quad-\infty<x<\infty, \\
\bar{\sigma}_{z L}(\zeta, 0)=\bar{\sigma}_{z}(\zeta, 0) \quad-\infty<x<\infty, \\
\bar{p}_{f}(\zeta, 0)=0 \quad-\infty<x<\infty, \\
\bar{\sigma}_{z L}\left(x,-H_{n}\right)=0 \quad|x|>b,
\end{gathered}
$$

where $\bar{\sigma}_{z}$ and $\bar{\tau}_{x z}$ are the normal stress and the shear stress of the soil skeleton, respectively; $\bar{p}_{f}$ is the pore pressure; $\bar{w}$ is the contact surface displacement between the strip foundation and underlying soil; $\phi$ is the rotation of the centre of the strip foundation; and $\bar{\Delta}(x)$ is the deflection of the strip foundation relative to the centre.

Combining (9a)-(9f) and (12a)-(12e) and applying the Fourier transform to (13b)-(13h), we can obtain the following relationships:

$$
\begin{aligned}
& 2 \bar{A}_{1} \zeta q_{L} H_{L} G_{L} \operatorname{sh}\left(-q_{L} H_{n}\right)+\bar{A}_{2}\left(\frac{F_{L}}{\zeta}+\frac{\zeta}{F_{L}}\right) G_{L} \operatorname{sh}\left(-F_{L} H_{n}\right) \\
& +2 \bar{B}_{1} \zeta q_{L} H_{L} G_{L} \operatorname{ch}\left(-q_{L} H_{n}\right) \\
& +\bar{B}_{2}\left(\frac{F_{L}}{\zeta}+\frac{\zeta}{F_{L}}\right) G_{L} \operatorname{ch}\left(-F_{L} H_{n}\right)=0 \\
& \quad \bar{B}_{1} q_{L} H_{L}+\frac{\bar{B}_{2}}{F_{L}}+A_{0} q H+B_{0} \zeta N+\frac{C_{0}}{F}=0 \\
& \quad-\bar{A}_{1} \zeta H_{L}-\frac{\bar{A}_{2}}{\zeta}+A_{0} \zeta H+B_{0} \zeta N+\frac{C_{0}}{\zeta}=0 \\
& \quad \bar{B}_{1} \zeta q_{L} H_{L} G_{L}+\bar{B}_{2}\left(\frac{F_{L}}{\zeta}+\frac{\zeta}{F_{L}}\right) G_{L}+2 A_{0} \zeta q H G \\
& +2 B_{0} \zeta^{2} N G+C_{0}\left(\frac{F}{\zeta}+\frac{\zeta}{F}\right) G=0
\end{aligned}
$$

$$
\begin{aligned}
& -2 G_{L}\left[\bar{A}_{1}\left(q_{L}^{2} H_{L}+\frac{\mu_{L}}{1-2 \mu_{L}}\right)+\bar{A}_{2}\right] \\
& +2 G\left[A_{0}\left(q^{2} H+\frac{\mu}{1-2 \mu}\right)+B_{0} \zeta^{2} N+C_{0}\right]-A_{0} E-B_{0}=0
\end{aligned}
$$

$$
A_{0} E+B_{0}=0
$$

From (14f) we can obtain

$$
B_{0}=-A_{0} E
$$

Utilising the Fourier transform on (12b) and (12e) gives

$$
\begin{aligned}
-2 G_{L}[ & \bar{A}_{1}\left(q_{L}^{2} H_{L}+\frac{\mu_{L}}{1-2 \mu_{L}}\right) \operatorname{ch}\left(-q_{L} H_{n}\right)+\bar{A}_{2} \operatorname{ch}\left(-F_{L} H_{n}\right) \\
& +\bar{B}_{1}\left(q_{L}^{2} H_{L}+\frac{\mu_{L}}{1-2 \mu_{L}}\right) \operatorname{sh}\left(-q_{L} H_{n}\right) \\
& \left.+\bar{B}_{2} \operatorname{sh}\left(-F_{L} H_{n}\right)\right]=\bar{\sigma}_{z}^{*}(\zeta, 0),
\end{aligned}
$$

$$
\begin{aligned}
& \bar{A}_{1} q_{L} H_{L} \operatorname{sh}\left(-q_{L} H_{n}\right)+\frac{\bar{A}_{2} \operatorname{sh}\left(-F_{L} H_{n}\right)}{F_{L}}+ \\
& \bar{B}_{1} q_{L} H_{L} \operatorname{ch}\left(-q_{L} H_{n}\right)+\frac{\bar{B}_{2} \operatorname{ch}\left(-F_{L} H_{n}\right)}{F_{L}}=\bar{w}^{*}(\zeta, 0) .
\end{aligned}
$$

Substituting (15) into (14b), (14c), (14d), and (14e), then (16a), (14a), (14b), (14c), (14d), and (14e) may be transformed into the following matrix form:

$$
\left[\begin{array}{llllll}
T_{11} & T_{12} & T_{13} & T_{14} & T_{15} & T_{16} \\
T_{21} & T_{22} & T_{23} & T_{24} & T_{25} & T_{26} \\
T_{31} & T_{32} & T_{33} & T_{34} & T_{35} & T_{36} \\
T_{41} & T_{42} & T_{43} & T_{44} & T_{45} & T_{46} \\
T_{51} & T_{52} & T_{53} & T_{54} & T_{55} & T_{56} \\
T_{61} & T_{62} & T_{63} & T_{64} & T_{65} & T_{66}
\end{array}\right] \cdot\left[\begin{array}{c}
\bar{A}_{1} \\
\bar{B}_{1} \\
\bar{A}_{2} \\
\bar{B}_{2} \\
A_{0} \\
C_{0}
\end{array}\right]=\left[\begin{array}{c}
\bar{\sigma}_{z}^{*}(\zeta, 0) \\
0 \\
0 \\
0 \\
0 \\
0
\end{array}\right] .
$$

The expression of each element of matrix $T$ can be seen in the appendix.

The displacement of the strip foundation surface can be expressed in the following matrix form:

$$
\begin{aligned}
& {\left[\begin{array}{llllll}
\Gamma_{1} & \Gamma_{2} & \Gamma_{3} & \Gamma_{4} & \Gamma_{5} & \Gamma_{6}
\end{array}\right]\left[\begin{array}{llllll}
\bar{A}_{1} & \bar{B}_{1} & \bar{A}_{2} & \bar{B}_{2} & A_{0} & C_{0}
\end{array}\right]^{T}} \\
& =\bar{w}^{*}(\zeta, 0),
\end{aligned}
$$


where

$$
\begin{gathered}
\Gamma_{1}=q_{L} H_{L} \operatorname{sh}\left(-q_{L} H_{n}\right), \\
\Gamma_{2}=q_{L} H_{L} \operatorname{ch}\left(-q_{L} H_{n}\right), \\
\Gamma_{3}=\frac{\operatorname{sh}\left(-F_{L} H_{n}\right)}{F_{L}}, \\
\Gamma_{4}=\frac{\operatorname{ch}\left(-F_{L} H_{n}\right)}{F_{L}}, \\
\Gamma_{5}=\Gamma_{6}=0 .
\end{gathered}
$$

Establishing the following equations by the matrix $T$ and the matrix $\Gamma$ gives

$$
T \cdot X=\Gamma,
$$

where $X$ is a $6 \times 1$ matrix.

We find from (17) and (18) that the element $X_{1}$ in the first row of the matrix $X$ denotes the relationship between the displacement and stress on the strip foundation surface. Thus, when

$$
\bar{w}^{*}(\zeta, 0)=f(\zeta) \cdot \bar{\sigma}_{z}^{*}(\zeta, 0)
$$

we obtain $f(\zeta)=X_{1}$, thereby expressing every element in matrix $X$ by solving (20). We can obtain $f(\zeta)$ and find that $f(\zeta)$ and $1 / \zeta$ are infinitesimal of the same order when $\zeta \rightarrow$ $\infty$.

Using the Fourier inverse transform and combining (21), we obtain

$$
\begin{aligned}
\bar{w}(x, 0) & =\frac{1}{2 \pi} \int_{-\infty}^{\infty} \bar{w}^{*}(\zeta, 0) e^{-i \zeta x} d \zeta \\
& =\frac{1}{\pi} \int_{0}^{\infty} f(\zeta) \bar{\sigma}_{z}^{*}(\zeta, 0) \sin (\zeta x) d \zeta .
\end{aligned}
$$

Additionally,

$$
\begin{aligned}
\bar{\sigma}_{z}(x, 0) & =\frac{1}{2 \pi} \int_{-\infty}^{\infty} \bar{\sigma}_{z}^{*}(\zeta, 0) e^{-i \zeta x} d \zeta \\
& =\frac{1}{\pi} \int_{0}^{\infty} \bar{\sigma}_{z}^{*}(\zeta, 0) \sin (\zeta x) d \zeta .
\end{aligned}
$$

The dual integral equations of the rocking vibration of an elastic strip foundation on elastic soil with saturated substrata are as follows:

$$
\begin{aligned}
& \int_{0}^{\infty} \tilde{f}(\widetilde{\zeta}) \tilde{\bar{\sigma}}_{z}^{*}(\widetilde{\zeta}, 0) \sin (\widetilde{\zeta} \tilde{x}) d \widetilde{\zeta} \\
& +\delta b^{2}\left(6 \tilde{x}^{3}-\tilde{x}^{2}\right) \\
& \times \int_{0}^{\infty} \frac{\left(1+\widetilde{\rho} \widetilde{h} \omega^{2} \tilde{f}(\widetilde{\zeta}) / G\right)}{\widetilde{\zeta}} \widetilde{\bar{\sigma}}_{z}^{*}(\widetilde{\zeta}, 0) \cos \widetilde{\zeta} d \widetilde{\zeta} \\
& -\delta b^{2} \tilde{x}^{2} \int_{0}^{\infty} \frac{\left(1+\tilde{\rho} \tilde{h} \omega^{2} \tilde{f}(\tilde{\zeta}) / G\right)}{\widetilde{\zeta}^{2}} \widetilde{\sigma}_{z}^{*}(\widetilde{\zeta}, 0) \sin \tilde{\zeta} d \tilde{\zeta} \\
& -\delta b^{2} \tilde{x} \int_{0}^{\infty} \frac{\left(1+\tilde{\rho} \tilde{h} \omega^{2} \tilde{f}(\tilde{\zeta}) / G\right)}{\widetilde{\zeta}^{3}} \widetilde{\bar{\sigma}}_{z}^{*}(\widetilde{\zeta}, 0) d \tilde{\zeta} \\
& -b^{2} \delta \int_{0}^{\infty} \frac{\left(1+\widetilde{\rho} \tilde{h} \omega^{2} \tilde{f}(\widetilde{\zeta}) / G\right)}{\widetilde{\zeta}^{4}} \widetilde{\bar{\sigma}}_{z}^{*}(\widetilde{\zeta}, 0)(\sin \tilde{\zeta} \widetilde{x}-2) d \widetilde{\zeta} \\
& =\pi x \phi b^{2} \quad 0 \leq|\widetilde{x}| \leq 1 \text {, } \\
& \int_{0}^{\infty} \widetilde{\bar{\sigma}}_{z}^{*}(\widetilde{\zeta}, 0) \sin (\widetilde{\zeta} \tilde{x}) d \widetilde{\zeta}=0 \quad|\widetilde{x}|>1,
\end{aligned}
$$

where $\phi$ is the rotation of the centre of the strip foundation and $D_{f}$ is the flexural stiffness of the foundation.

Simpson's rule is used to conduct numerical calculation. The dynamic compliance coefficient, $C_{M}$, of the rocking vibration of a strip foundation can be expressed as follows [27]:

$$
C_{M}=\frac{1}{b a_{0}} .
$$

Defining $f_{1}=\operatorname{Re}\left[C_{M}\right]$ and $f_{2}=\operatorname{Im}\left[C_{M}\right]$, we can obtain foundation stiffness $K=f_{1} /\left(f_{1}^{2}+f_{2}^{2}\right)$ and the damping coefficient of the foundation $C=-f_{2} /\left(f_{1}^{2}+f_{2}^{2}\right) b_{0}$. Here, $b_{0}=b \omega \sqrt{\rho / G}$ is the dimensionless frequency.

\section{Verifications and Numerical Example Analysis}

The rocking vibration solution of an elastic strip foundation on elastic soil with saturated substrata can be degenerated to the single-phase elastic half-space case by defining $\rho_{f}=0$, $\delta=0$, and $H_{n}=0$. The foundation parameters for the degenerated case are $b=2 \mathrm{~m}, G=35 \mathrm{MPa}, n=0.35$, and $\rho_{s}=2650 \mathrm{~kg} / \mathrm{m}^{3}$. The variation of the dynamic compliance coefficient $C_{M}$ with dimensionless frequency $b_{0}$ is analysed and is then compared with the numerical results by Luco and Westmann [5] when $\mu$ is 0.25 . In Figure 2, "o" represents the numerical results obtained by Luco and Westmann [5] when $\mu$ is 0.25 . Both of the results derived from Figure 2 are consistent and verify the feasibility and accuracy of the calculating methods described in this paper. Meanwhile, the rigid foundation is considered as a special case of the elastic foundation when $\delta$ equals zero. 


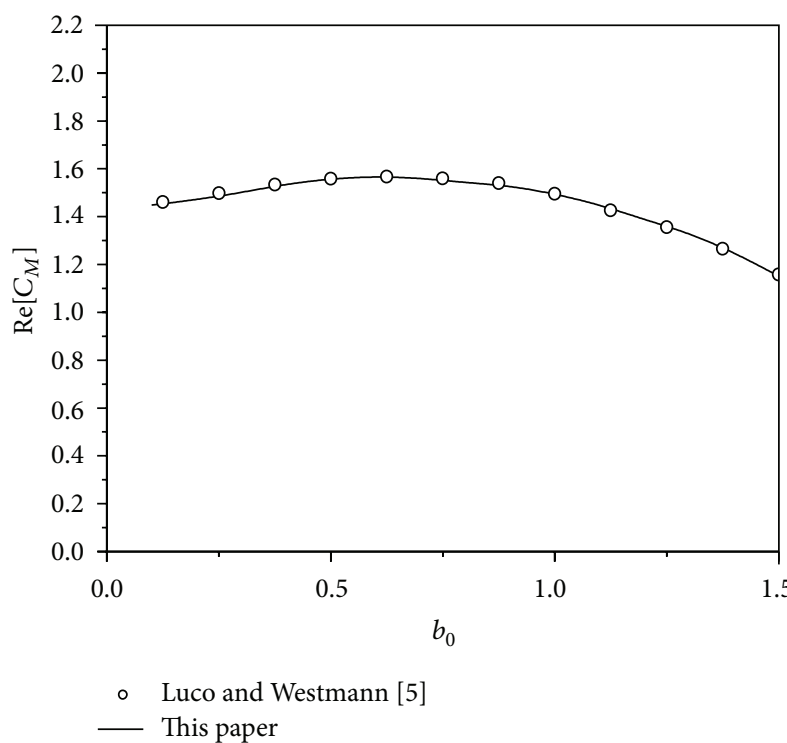

(a)

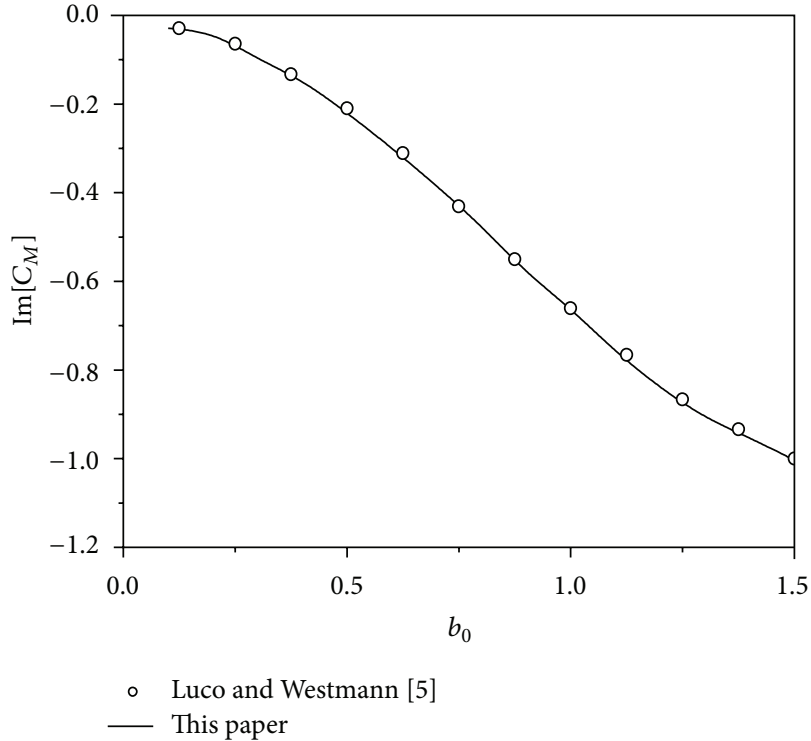

(b)

FIgURE 2: The dynamic compliance coefficient versus the dimensionless frequency. (a) Real part of $C_{M}$ versus the dimensionless frequency and (b) imaginary part of $C_{M}$ versus the dimensionless frequency.

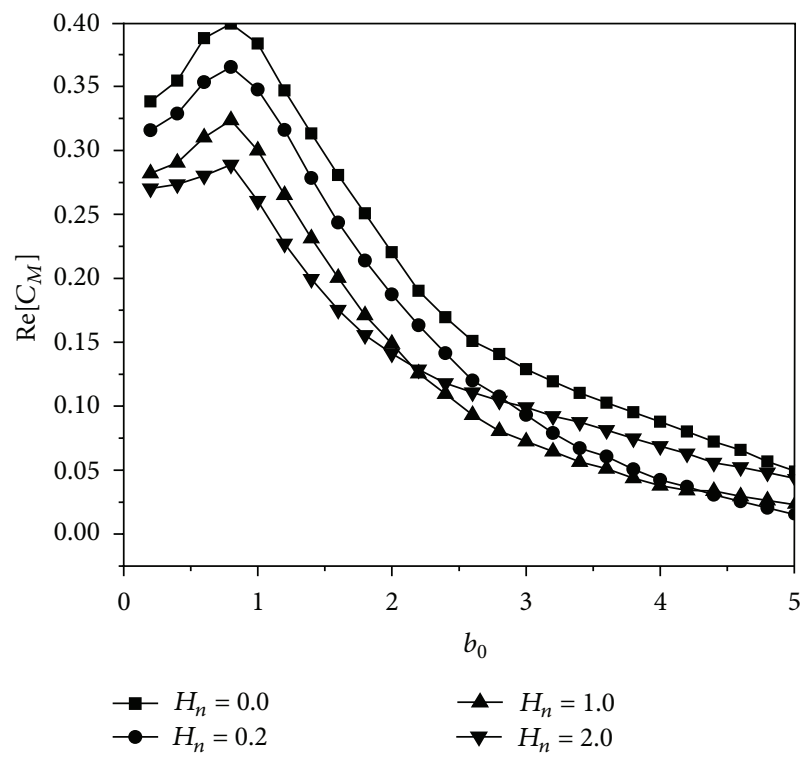

(a)

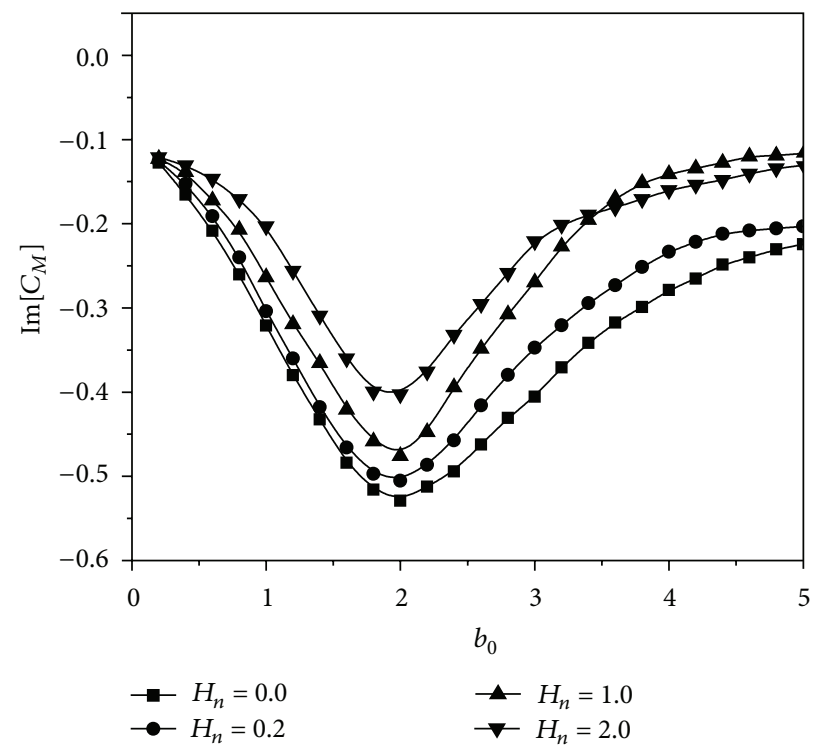

(b)

Figure 3: The dynamic compliance coefficient $C_{M}$ of different single-phase elastic layer thicknesses $\left(H_{n}\right)$. (a) Real part of $C_{M}$ versus the dimensionless frequency and (b) imaginary part of $C_{M}$ versus the dimensionless frequency.

Concerning the rocking vibration of an elastic strip foundation on elastic soil with saturated substrata, the physical and mechanical parameters of a single-phase elastic layer are $G_{L}=35 \mathrm{MPa}, \mu_{L}=0.45$, and $\rho_{L}=1722.5 \mathrm{~kg} / \mathrm{m}^{3}$; for a saturated half-space, $n=0.35, G=35 \mathrm{MPa}, k_{d}=10^{-5} \mathrm{~m} / \mathrm{s}$, $\rho_{f}=1000 \mathrm{~kg} / \mathrm{m}^{3}, \rho_{s}=2650 \mathrm{~kg} / \mathrm{m}^{3}$, and $\mu=0.25$. The dynamic compliance coefficient $C_{M}$ changes over the dimensionless frequency, the state of which is calculated when
$H_{n}=0,0.2,1.0$, and $2.0 \mathrm{~m}$ for $\delta=10$, and the calculation results are shown in Figure 3. Meanwhile, the dynamic compliance coefficient $C_{M}$ changes over the dimensionless frequency, the state of which is calculated when $\delta=0.0,0.1,10$, and 1000 for $H_{n}=0.2$, and the calculation results are shown in Figure 4.

We can see from Figure 3 that $C_{M}$ decreases with an increase in the elastic layer thickness, which indicates that 


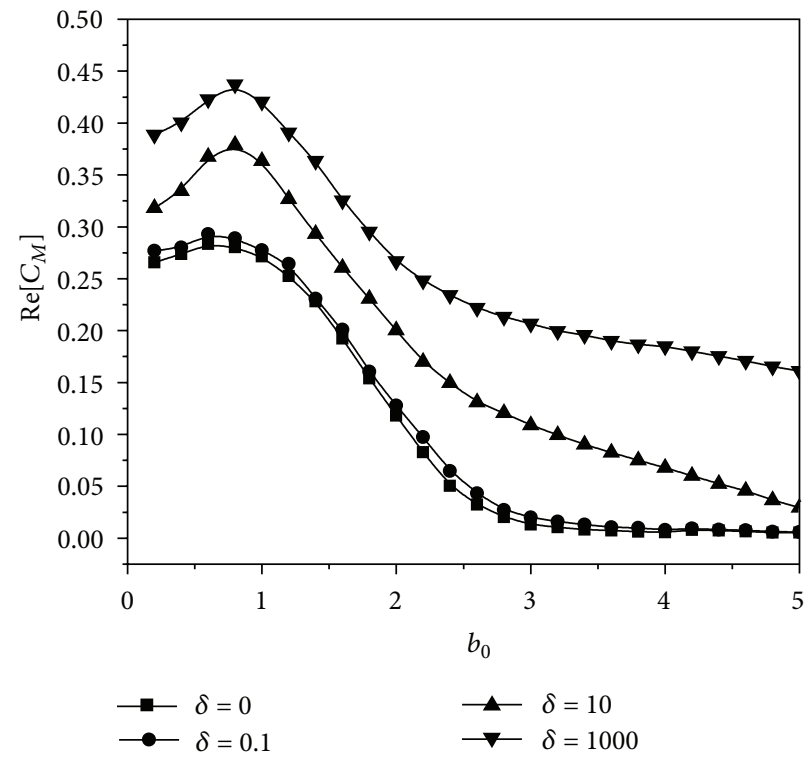

(a)

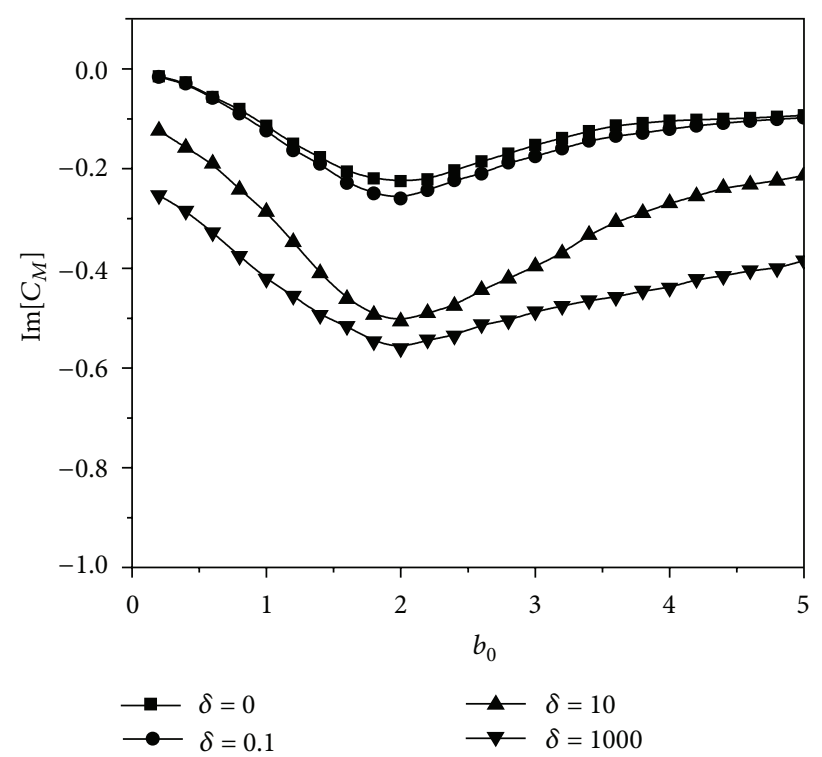

(b)

FIgURE 4: The dynamic compliance coefficient $C_{M}$ for different $\delta$. (a) Real part of $C_{M}$ versus the dimensionless frequency and (b) imaginary part of $C_{M}$ versus the dimensionless frequency.

the presence of elastic soil can reduce the vibration to the foundation. Meanwhile, in the given parameters for the real part of $C_{M}$, as $b_{0}$ increases, the real part of $C_{M}$ decreases, but the curve tends to flatten. For the imaginary part of $C_{M}$, as $b_{0}$ increases, the imaginary part of $C_{M}$ first decreases and then increases, finally becoming smooth.

For the rocking vibration of an elastic strip foundation on elastic soil with saturated substrata, the curves of $C_{M}$ are essentially coincident when $\delta=0.0$ and 0.1 , which can be seen from Figure 4. The curve for $\delta=0.0$ is the one of the dynamic compliance coefficients of the rocking vibration of a rigid strip foundation on elastic soil with saturated substrata. Therefore, it can be inferred that the rocking vibration of an elastic and a rigid strip foundation on elastic soil with saturated substrata has the similar dynamic characteristics in variations of $b_{0}$ when $\delta \leq 0.1$. It is also demonstrated that the real part of $C_{M}$ is greatly influenced by variations in $b_{0}$ when $b_{0}<2.8$ and that the imaginary part of $C_{M}$ is greatly influenced by variations in $b_{0}$ when $b_{0}<3.6$. However, when $b_{0}$ exceeds the critical values (2.8 for the real part of $C_{M}$ and 3.6 for the imaginary part of $C_{M}$ ), the curve of $C_{M}$ tends to flatten and $C_{M}$ is only slightly influenced by variations in the dimensionless frequency $b_{0}$. Figure 4 also shows that the dynamic compliance coefficient curve obtained when $\delta=10$ and 1000 is remarkably different from the one obtained when $\delta=0.0$ and 0.1 , and the absolute values concerning both the real parts and imaginary parts of the dynamic compliance coefficient when $\delta=10$ and 1000 are larger than the ones when $\delta=0.0$ and 0.1. Moreover, it can be seen from Figure 4 that when $\delta$ is large, the variation of the dynamic compliance coefficient curve with the dimensionless frequency $b_{0}$ tends to be smooth. However, for an average quantity of $\delta$, the variation of the absolute values of the real parts and imaginary parts of $C_{M}$ with $b_{0}$ is significant. Thus, under ordinary circumstances $(\delta=10)$, we must consider effects exerted by the dimensionless frequency $b_{0}$.

\section{Conclusions}

In this paper, an analytical solution for the rocking vibration of an elastic strip foundation on elastic soil with saturated substrata is developed. The solution is based on dual integral equations, which are formulated from Biot's equations of dynamic poroelasticity by means of the Fourier transform in combination with mixed boundary conditions. Validation of the analytical solution for dry soil is based on the solution presented by Luco and Westmann [5].

Our conclusions of this study are as follows. (1) The dynamic compliance coefficient $C_{M}$ decreases with an increase in the elastic layer thickness, which indicates that the presence of elastic soil can reduce the vibration to the foundation. (2) The real part of dynamic compliance coefficient $C_{M}$ is greatly influenced by variations in the dimensionless frequency $b_{0}$ when $b_{0}<2.8$, and the imaginary part of $C_{M}$ is greatly influenced by variations in the dimensionless frequency $b_{0}$ when $b_{0}<3.6$. (3) When the flexural stiffness of the elastic foundation is comparatively large or when $\delta \leq 0.1$, the influence of $\delta$ on the rocking vibration can be ignored. (4) When $\delta>0.1$ and as $\delta$ increases, the rocking vibration of the elastic foundation changes significantly, and the absolute values of both the real parts and imaginary parts of $C_{M}$ increase. 


\section{Appendix}

Expressions for each element of the matrix $T$ appearing previously are given by

$$
\begin{aligned}
& T_{11}=-2 G_{L}\left(q_{L}^{2} H_{L}+\frac{\mu_{L}}{1-2 \mu_{L}}\right) \operatorname{ch}\left(-q_{L} H_{n}\right), \\
& T_{12}=-2 G_{L}\left(q_{L}^{2} H_{L}+\frac{\mu_{L}}{1-2 \mu_{L}}\right) \operatorname{sh}\left(-q_{L} H_{n}\right) \text {, } \\
& T_{13}=-2 G_{L} \operatorname{ch}\left(-F_{L} H_{n}\right), \\
& T_{14}=-2 G_{L} \operatorname{sh}\left(-F_{L} H_{n}\right) \text {, } \\
& T_{15}=0 \text {, } \\
& T_{16}=0 \text {, } \\
& T_{21}=2 \zeta q_{L} H_{L} G_{L} \operatorname{sh}\left(-q_{L} H_{n}\right), \\
& T_{22}=2 \zeta q_{L} H_{L} G_{L} \operatorname{ch}\left(-q_{L} H_{n}\right), \\
& T_{23}=\left(\frac{F_{L}}{\zeta}+\frac{\zeta}{F_{L}}\right) G_{L} \operatorname{sh}\left(-F_{L} H_{n}\right) \text {, } \\
& T_{24}=\left(\frac{F_{L}}{\zeta}+\frac{\zeta}{F_{L}}\right) G_{L} \operatorname{ch}\left(-F_{L} H_{n}\right), \\
& T_{25}=0 \text {, } \\
& T_{26}=0 \text {, } \\
& T_{31}=0, \\
& T_{32}=q_{L} H_{L}, \\
& T_{33}=0 \text {, } \\
& T_{34}=\frac{1}{F_{L}}, \\
& T_{35}=q H-\zeta N E, \\
& T_{36}=\frac{1}{F}, \\
& T_{41}=-\zeta H_{L}, \\
& T_{42}=0 \text {, } \\
& T_{43}=-\frac{1}{\zeta}, \\
& T_{44}=0 \text {, } \\
& T_{45}=\zeta H-\zeta N E, \\
& T_{46}=\frac{1}{\zeta}, \\
& T_{51}=0 \text {, } \\
& T_{52}=2 \zeta q_{L} H_{L} G_{L} \text {, } \\
& T_{53}=0 \text {, }
\end{aligned}
$$

$$
\begin{gathered}
T_{54}=\left(\frac{F_{L}}{\zeta}+\frac{\zeta}{F_{L}}\right) G_{L}, \\
T_{55}=2 \zeta q H G-2 \zeta^{2} N G E, \\
T_{56}=\left(\frac{F}{\zeta}+\frac{\zeta}{F}\right) G, \\
T_{61}=-2 G_{L}\left(q_{L}^{2} H_{L}+\frac{\mu_{L}}{1-2 \mu_{L}}\right), \\
T_{62}=0, \\
T_{63}=-2 G_{L}, \\
T_{64}=0, \\
T_{65}=2 G\left[\left(q^{2} H+\frac{\mu}{1-2 \mu}\right)-\zeta^{2} N E\right], \\
T_{66}=2 G .
\end{gathered}
$$

\section{Acknowledgment}

The work presented in this paper is partly supported by the National Natural Science Foundation of China (Grant nos. 51179171, 51079127, and 51279180).

\section{References}

[1] T. Y. Sung, "Vibrations in semi-infinite solids due to periodic surface loadings," in Symposium on Dynamic Testing of Soils, ASTM Special Technical Publication, no.156, pp. 35-64, 1953.

[2] A. O. Awojobi, "Harmonic rocking of a rigid rectangular body on a semi-infinite elastic medium," Journal of Applied Mechanics, vol. 33, no. 3, pp. 547-552, 1966.

[3] H. S. Paul, "Vibration of a rigid circular disk on an infinite isotropic elastic plate," The Journal of Acoustical Society of America, vol. 42, no. 2, pp. 412-416, 1967.

[4] M. A. Oien, "Steady motion of a rigid strip bonded to an elastic half space," Journal of Applied Mechanics, vol. 38, no. 2, pp. 328334, 1971.

[5] J. E. Luco and R. A. Westmann, "Dynamic response of a rigid footing bounded to an elastic half space," Journal of Applied Mechanics, vol. 39, no. 2, pp. 527-534, 1972.

[6] X. Zeng and A. S. Cakmak, "Dynamic structure-soil interaction under the action of vertical ground motion, Part II: the plane problem," International Journal of Soil Dynamics and Earthquake Engineering, vol. 3, no. 4, pp. 211-219, 1984.

[7] M. A. Biot, "Theory of propagation of elastic waves in a fluidsaturated porous solid. I. Low-frequency range," The Journal of the Acoustical Society of America, vol. 28, pp. 168-178, 1956.

[8] M. A. Biot, "Theory of propagation of elastic waves in a fluidsaturated porous solid. II. Higher frequency range," The Journal of the Acoustical Society of America, vol. 28, no. 2, pp. 179-191, 1956.

[9] Y. J. Lin, "Dynamic response of circular plates resting on viscoelastic half space," Journal of Applied Mechanics, vol. 45, no. 2, pp. 379-384, 1978. 
[10] M. Iguchi and J. E. Luco, "Vibration of flexible plate on viscoelastic medium," Journal of the Engineering Mechanics Division, vol. 108, no. 6, pp. 1103-1120, 1982.

[11] M. R. Halpern and P. Christiano, "Response of poroelastic halfspace to steady-state harmonic surface tractions," International Journal for Numerical \& Analytical Methods in Geomechanics, vol. 10, no. 6, pp. 609-632, 1986.

[12] M. R. Halpern and P. Christiano, "Steady-state harmonic response of a rigid plate bearing on a liquid-saturated poroelastic halfspace," Earthquake Engineering \& Structural Dynamics, vol. 14, no. 3, pp. 439-454, 1986.

[13] M. K. Kassir and J. M. Xu, "Interaction functions of a rigid strip bonded to saturated elastic half-space," International Journal of Solids and Structures, vol. 24, no. 9, pp. 915-936, 1988.

[14] B. Jin and H. Liu, "Horizontal vibrations of a disk on a poroelastic half-space," Soil Dynamics and Earthquake Engineering, vol. 19, no. 4, pp. 269-275, 2000.

[15] B. Jin and H. Liu, "Rocking vibrations of rigid disk on saturated poroelastic medium," Soil Dynamics and Earthquake Engineering, vol. 19, no. 7, pp. 469-472, 2000.

[16] Q. J. Li, Vertical vibration analysis of strip foundation on saturated soils [M.S. thesis], Zhejiang University, China, 2005.

[17] X. H. Ma, Y. M. Cheng, S. K. Au, Y. Q. Cai, and C. J. Xu, "Rocking vibration of a rigid strip footing on saturated soil," Computers and Geotechnics, vol. 36, no. 6, pp. 928-933, 2009.

[18] A. J. Philippacopoulos, "Axisymmetric vibration of disk resting on saturated layered half-space," Journal of Engineering Mechanics, vol. 115, no. 10, pp. 2301-2322, 1989.

[19] S. Bougacha, J. L. Tassoulas, and J. M. Roesset, "Analysis of foundations on fluid-filled poroelastic stratum," Journal of Engineering Mechanics, vol. 119, no. 8, pp. 1632-1648, 1993.

[20] S. Bougacha, J. M. Roesset, and J. L. Tassoulas, "Dynamic stiffness of foundations on fluid-filled poroelastic stratum," Journal of Engineering Mechanics, vol. 119, no. 8, pp. 1649-1662, 1993.

[21] R. K. N. D. Rajapakse and T. Senjuntichai, "Dynamic response of a multi-layered poroelastic medium," Earthquake Engineering \& Structural Dynamics, vol. 24, no. 5, pp. 703-722, 1995.

[22] J. Yang, S. M. Wu, and Y. M. Chen, "Steady state response of elastic soil layer and saturated layered half-space," China Civil Engineering Journal, vol. 30, no. 3, pp. 39-48, 1997.

[23] O. C. Zienkiewicz, C. T. Chang, and P. Bettess, "Drained, undrained, consolidating and dynamic behaviour assumptions in soils," Geotechnique, vol. 30, no. 4, pp. 385-395, 1980.

[24] S. L. Chen, Study on the vertical vibrations of foundations testing on saturated grounds [Ph.D. thesis], Zhejiang University, China, 2000.

[25] G. C. Wang, Study on the torsional vibrations of foundations resting on saturated grounds [Ph.D. thesis], Zhejiang University, China, 2002.

[26] B. Fu, Study on the rocking vibrations of foundations resting on saturated grounds [Ph.D. thesis], Zhejiang University, China, 2005.

[27] R. J. Yan, Y. S. Wang, and Q. Y. Han, The Theory of Dynamic Response of the Footing with A half space, China Architecture and Building Press, Beijing, China, 1981. 


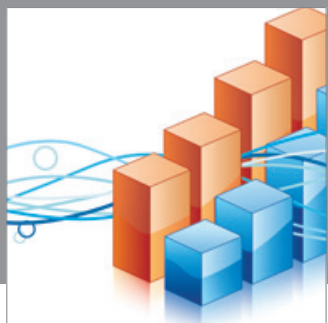

Advances in

Operations Research

mansans

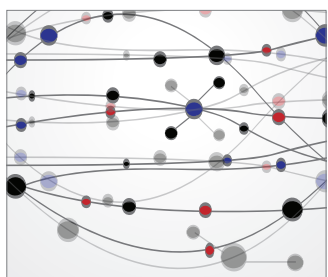

The Scientific World Journal
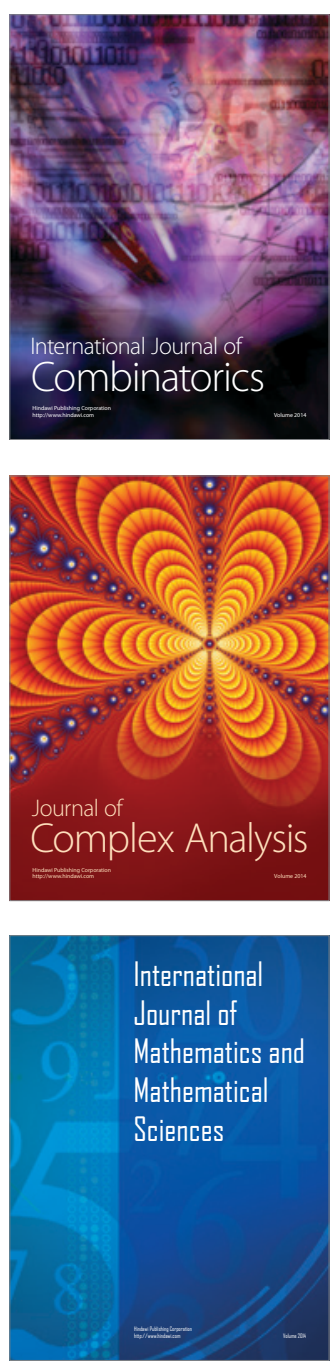
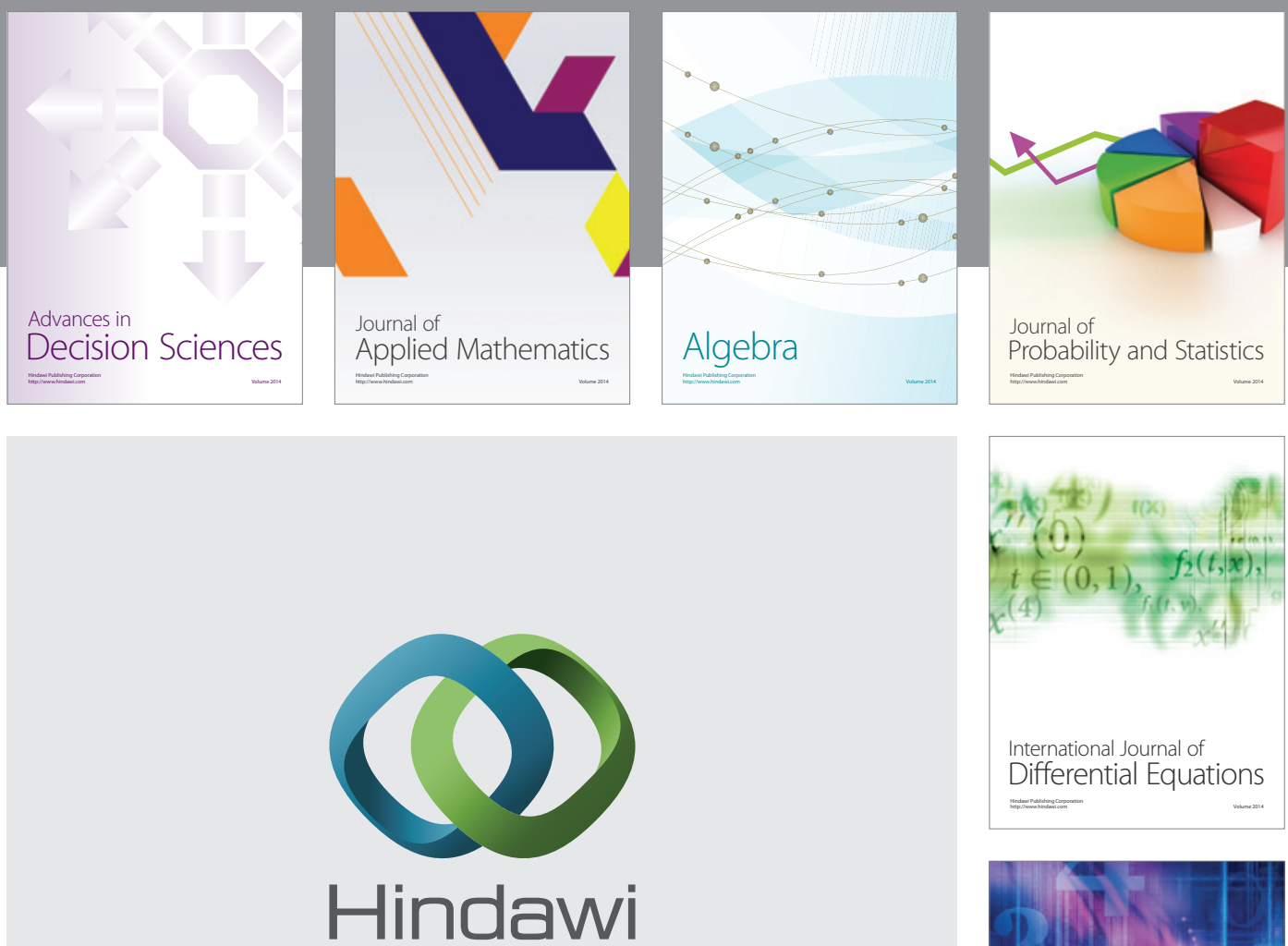

Submit your manuscripts at http://www.hindawi.com
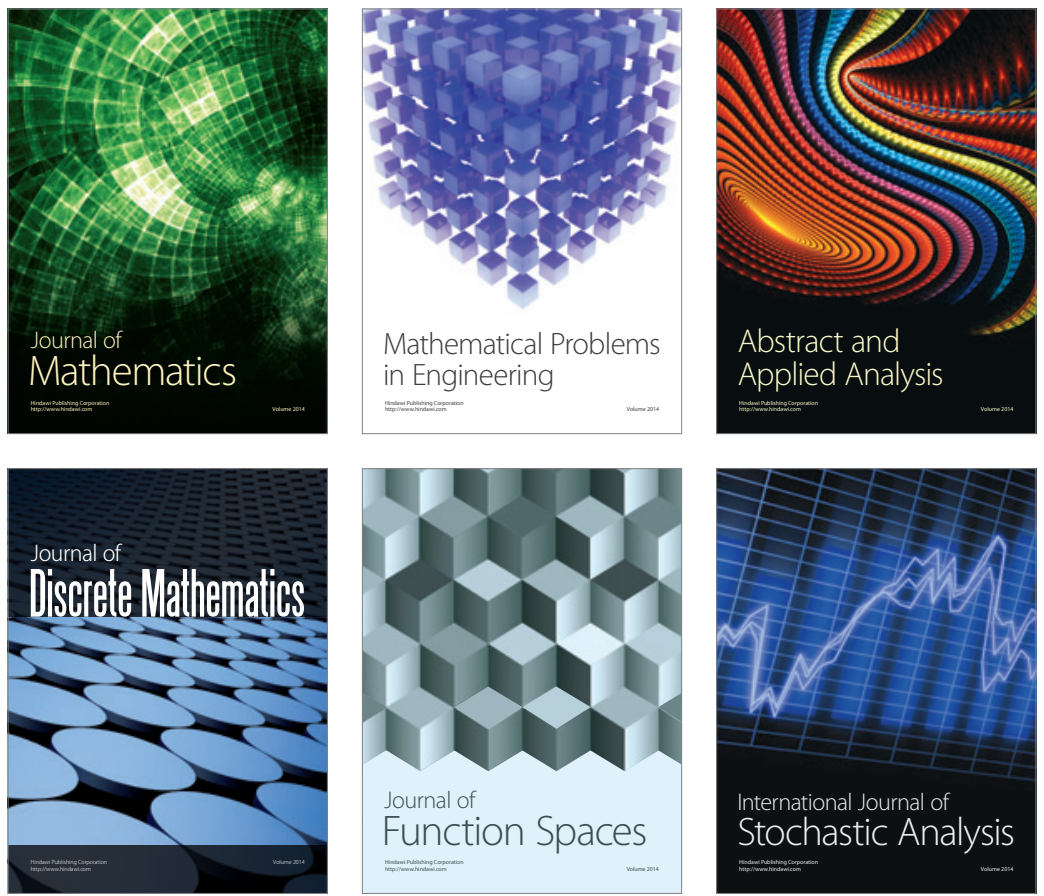

Journal of

Function Spaces

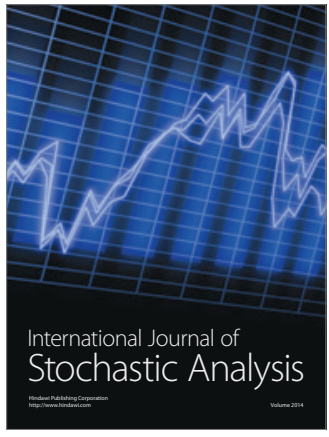

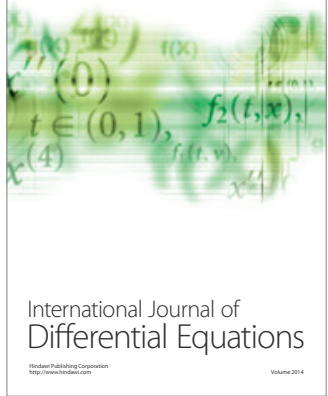
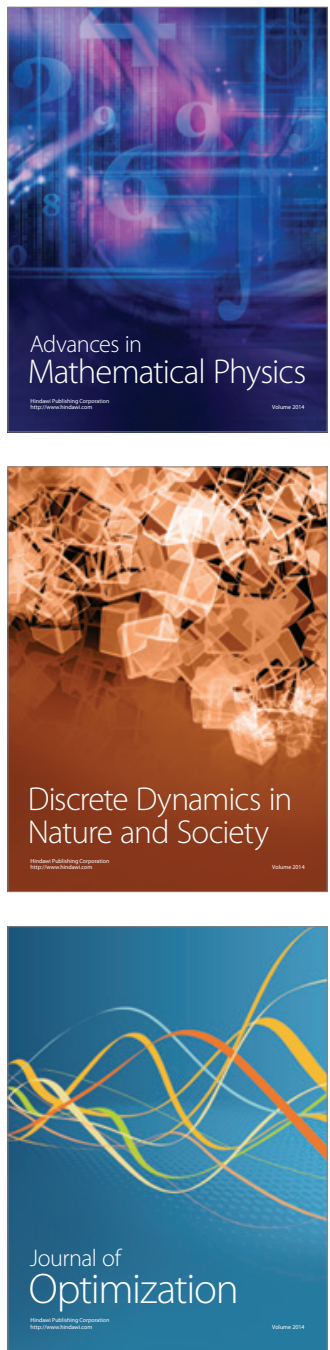